\title{
MANIFEST instrument concept and related technologies
}

\author{
Michael Goodwin $^{* 1}$, Jurek Brzeski ${ }^{1}$, Scott Case ${ }^{1}$, Matthew Colless ${ }^{1}$, Tony Farrell ${ }^{1}$, Luke Gers ${ }^{1}$, \\ James Gilbert ${ }^{1}$, Jeroen Heijmans ${ }^{1}$, Andrew Hopkins ${ }^{1}$, Jon Lawrence ${ }^{1}$, Stan Miziarski ${ }^{1}$, Guy \\ Monnet $^{1,2}$, Rolf Muller $^{1}$, Will Saunders ${ }^{1}$, Greg Smith ${ }^{1}$, Julia Tims $^{1}$, Lew Waller ${ }^{1}$ \\ 1. Australian Astronomical Observatory, PO Box 296 Epping, NSW 1710, Australia \\ 2. Current address: Observatoire de Lyon, 9 avenue Charles André, 69561 Saint Genis Laval Cedex, France
}

\begin{abstract}
The Australian Astronomical Observatory (AAO) has recently completed a feasibility study for a fiber-positioner facility proposed for the Giant Magellan Telescope (GMT), called MANIFEST (the Many Instrument Fiber System). The MANIFEST facility takes full advantage of the wide-field focal plane to efficiently feed a number of focal instruments. It is a simple, flexible and modular design, based on the AAO's experience and R\&D with starbugs, robotic positioners, and related fiber technologies for astronomical instrumentation. Up to 2000 individually deployable fiber units are envisaged, with a wide variety of aperture types (single-aperture, image- or pupil-slicing, IFU). MANIFEST allows (a) full use of the GMT's 20' field-of-view, (b) a multiplexed IFU capability, (c) greatly increased spectral resolution via image-slicing, (d) the possibility of OH-suppression in the near-infrared. It is intended that MANIFEST will form part of the GMT facility itself, available to any instrument able to make use of it. In this paper, we report on the recent progress involving the science goals, instrument concept, related technologies and performances.
\end{abstract}

Keywords: fiber positioners, starbugs, extremely large telescopes, $\mathrm{OH}$ suppression

\section{INTRODUCTION}

The AAO has a strong track record in delivering successful fiber technologies and robotic positioners for astronomy, as demonstrated with the $2 \mathrm{dF}^{1}$, FLAMES/OzPoz ${ }^{2}$ and FMOS/Echidna ${ }^{19}$ instruments. As such, the AAO has proposed a concept for the GMT $^{3}$ Facility Multi-Object Fiber System (GFFS), called MANIFEST, the Many Instrument Fiber System $^{4}$. MANIFEST is a fiber feed system for GMT that offers access to larger fields of view, higher multiplex gains, versatile reformatting of the focal plane via IFUs and image-slicers, and in some cases higher spatial and spectral resolution. Coupled to the proposed seeing-limited instruments GMACS $^{5}, \mathrm{NIRMOS}^{6} \& \mathrm{G}^{-\mathrm{CLEF}^{7}}$, it provides qualitative and quantitative gains over each instrument's standalone capabilities in terms of both instrumental parameter space coverage and scientific grasp and performance. The potential benefits to the GMT are as follows:

World-leading capabilities: MANIFEST is not only a major force multiplier for GMT instruments and science; it also provides GMT with world-leading capabilities compared to the other Extremely Large Telescopes (ELTs). Although GMT is the ELT with the smallest aperture (A), it has the equal-largest field of view $(\Omega)$. It cannot beat the other ELTs at science where the figure of merit is the primary mirror diameter (D) to powers greater than unity (so-called $\mathrm{D}^{\mathrm{n}}$ science). However it can dominate wide-field and survey astronomy (so-called $A \Omega$ science), because the other ELTs are not making use of the full field of view delivered by the telescope. This comparison is made explicit in Table 1 below, which shows that GMT is at a serious disadvantage for $\mathrm{D}^{2}$ and (a fortiori) $\mathrm{D}^{4}$ science, but that it can have a substantial advantage (a factor 2.5 to 4 ) in terms of $A \Omega$ provided its instruments can access the full field of view offered by the telescope.

mgoodwin@aao.gov.au; http://www.aao.gov.au;

phone +6129372 4851; fax +61293724860

Ground-based and Airborne Instrumentation for Astronomy IV, edited by lan S. McLean, Suzanne K. Ramsay, Hideki Takami, Proc. of SPIE Vol. 8446, 84467I · @ 2012 SPIE · CCC code: 0277-786X/12/\$18 · doi: 10.1117/12.925125 
Table 1: Comparison of ELTs and their survey spectroscopy capabilities

\begin{tabular}{|l|l|l|l|l|l|l|l|}
\hline Telescope+Instrument & Diam.(D) & Tel.Field $\left(\phi_{\mathrm{T}}\right)$ & Inst.Field $\left(\Omega_{\mathrm{I}}\right)$ & $\mathrm{D}^{2}$ & $\mathrm{D}^{4}$ & ${ }^{+} \mathrm{A} \Omega_{\mathrm{T}}$ & ${ }^{+} \mathrm{A} \Omega_{\mathrm{I}}$ \\
\hline GMT+MANIFEST & $25.4 \mathrm{~m}$ & $20^{\prime}$ & $314^{\prime}$ & $\equiv 1$ & $\equiv 1$ & $\equiv 1$ & $\equiv 1$ \\
\hline TMT+WFOS $^{8}$ & $30 \mathrm{~m}$ & $20^{\prime}$ & $40.5^{\prime}$ & 1.4 & 1.9 & 1.78 & 0.23 \\
\hline E-ELT+DIORAMAS $^{9}$ & $39.3 \mathrm{~m}$ & $10^{\prime}$ & $46^{\prime}$ & 2.4 & 5.7 & 0.66 & 0.39 \\
\hline
\end{tabular}

${ }^{+}$Note: collecting area of telescopes GMT $=368, \mathrm{TMT}=655, \mathrm{E}-\mathrm{ELT}=978 \mathrm{~m}^{2}$

Note that in this comparison the range in field of view for TMT+WFOS depends on whether WFOS is a two-shooter or a four-shooter, but GMT+MANIFEST can access the full telescope field of view regardless of the number of GMACS arms (reduced multiplex but field of view unchanged). Moreover the table flatters TMT and E-ELT because the comparison applies to their widest-field instruments, whereas MANIFEST can provide the full GMT field of view to any spectroscopic instrument with a sizable spatial coverage. With MANIFEST, GMT has the largest effective A $\Omega$ product and will therefore be the leading ELT for any type of wide-field or survey astronomy.

Enhanced functionality: MANIFEST offers GMT spectrographs a wide range of enhanced functionality: (1) increased fields of view; (2) multiple deployable IFUs; (3) increased resolution via image-slicing; (4) efficient detector packing, both spectrally and spatially; (5) efficiency gains from working at VPHG super-blaze angles; (6) simultaneous observations with multiple instruments; (7) gravity-invariant spectrograph mounting; and (8) OH suppression in the NIR. Capabilities such as the deployable IFUs, increased multiplex and increased resolution enable entirely new science with GMT; other functionality offers efficiency gains ranging from incremental (e.g. efficient detector packing and working at the superblaze angle), to substantial (increased fields of view and simultaneous observations), to transformative (multiIFUs and OH suppression). MANIFEST easily exceeds the requirements for the GFFS facility.

Versatility: The MANIFEST concept increases the versatility of GMT by embodying three principles in its technical design - (1) selectability: instruments can work either in their native mode or with fiber feeds that reformat the focal plane in a variety of ways; (2) modularity: new instruments can easily be accommodated by adding new fiber modules; and (3) upgradeability: the system can provide new functionality via straightforward and relatively low cost upgrades of the fiber modules. MANIFEST is not an instrument but rather an integrated telescope facility, analogous to adaptive optics systems in providing broad scientific utility and enhanced performance for a variety of instruments. MANIFEST expands the accessible parameter space and enhances the long-term potentialities of GMT's instrument suite.

The AAO remains fully committed to the development of MANIFEST with the 2011 completion of its Feasibility Study and continual ongoing R\&D efforts on related enabling technologies. The key areas addressed in the Feasibility Study report are to: a) retire major technical risks with the design of MANIFEST; b) estimate its cost versus added functional and scientific value, and c) establish the interface requirements with the telescope and the GMACS, G-CLEF \& NIRMOS instruments by liaising with GMTO and the relevant instrument teams. All of these goals were addressed in the Feasibility Study report. The main technological risks have been retired through design and prototyping studies; the functional and scientific added value of the baseline instrument design have been determined.

This paper documents the key outcomes of the Feasibility Study and the R\&D efforts that have advanced the MANIFEST concept as a prospective facility for the GMT. Section 2 summarizes the science goals. Section 3 reports on the instrument concept. Section 4 reports on the related technology R\&D. Section 5 summarizes the predicted throughput performance. Concluding remarks are provided in Section 6. 


\section{SCIENCE GOALS}

In this section we provide an updated summary of the science goals of MANIFEST. MANIFEST significantly increases the versatility and capability of GMT instruments, greatly enlarging the accessible discovery space. This affects the GMT science cases in three ways: (a) for some cases it provides substantial quantitative gains, allowing programs to be carried out faster or better; (b) in some cases it provides a major leap in performance, allowing new experiments to be undertaken, and (c) in some cases, it provides an entirely new capability.

Quantitative Gains: Systematic surveys of wide areas of sky and/or large numbers of objects are becoming more and more important in astronomy. For such survey (or $A \Omega$ ) astronomy, the crucial criterion is overall survey sensitivity: observing the largest number of targets, with the least background, at the highest throughput and observing efficiency, over the widest FOV. In this context, the gains from MANIFEST are best quantified in terms of the 'survey speed' figure of merit. Survey speed is the inverse of the time taken to observe targets of a given brightness and surface density, over a given area of sky, to a given signal-to-noise ratio. Table 2 shows the approximate gains in survey speed that MANIFEST provides for GMACS, NIRMOS and G-CLEF relative to their native performance.

Table 2: Gains in survey speed provided by MANIFEST for GMT instruments

\begin{tabular}{|c|c|c|}
\hline Instrument & Field-limited gain & Multiplex-limited gain \\
\hline GMACS & $\sim 2$ & $\sim 2$ \\
\hline NIRMOS & $\sim 4$ & $\sim 2$ \\
\hline G-CLEF all orders & $\sim 4$ & $\sim 4$ \\
\hline G-CLEF few orders & $\sim 43$ & $\sim 43$ \\
\hline
\end{tabular}

Qualitative Gains: The baseline MANIFEST design offers qualitative gains for GMT science in a number of areas, including image-slicing (giving increased spectral resolution), multi-IFUs (spatially resolved area spectroscopy) and single-target $\mathrm{OH}$-suppression (large reductions in the near-infrared sky background), as well as straightforward ways to expand the multiplex gain as larger detectors become available. Other future upgrades to MANIFEST offer further qualitative gains, such as high-multiplex $\mathrm{OH}$-suppression for large deep near-infrared surveys and multi-object adaptive optics for multiplex area spectroscopy at high spatial resolution.

The capabilities of the various modes offered in the baseline MANIFEST concept, and their application to the key GMT science $\operatorname{cases}^{20}$, are illustrated schematically in Figure 1; a comprehensive summary of the various modes in terms of format, multiplex, spatial sampling, field of view and spectral resolution is provided in Table 3 . The lower left box lists the key programs in the GMT Science Case and the GFFS RFP. The box at lower right shows schematically the various modes offered by MANIFEST for each of GMACS, NIRMOS and G-CLEF. The top panel shows the relation of the science cases to the MANIFEST modes; for each of the science cases, color-coding shows the corresponding instruments, while the numbered icons show the relevant MANIFEST modes. 


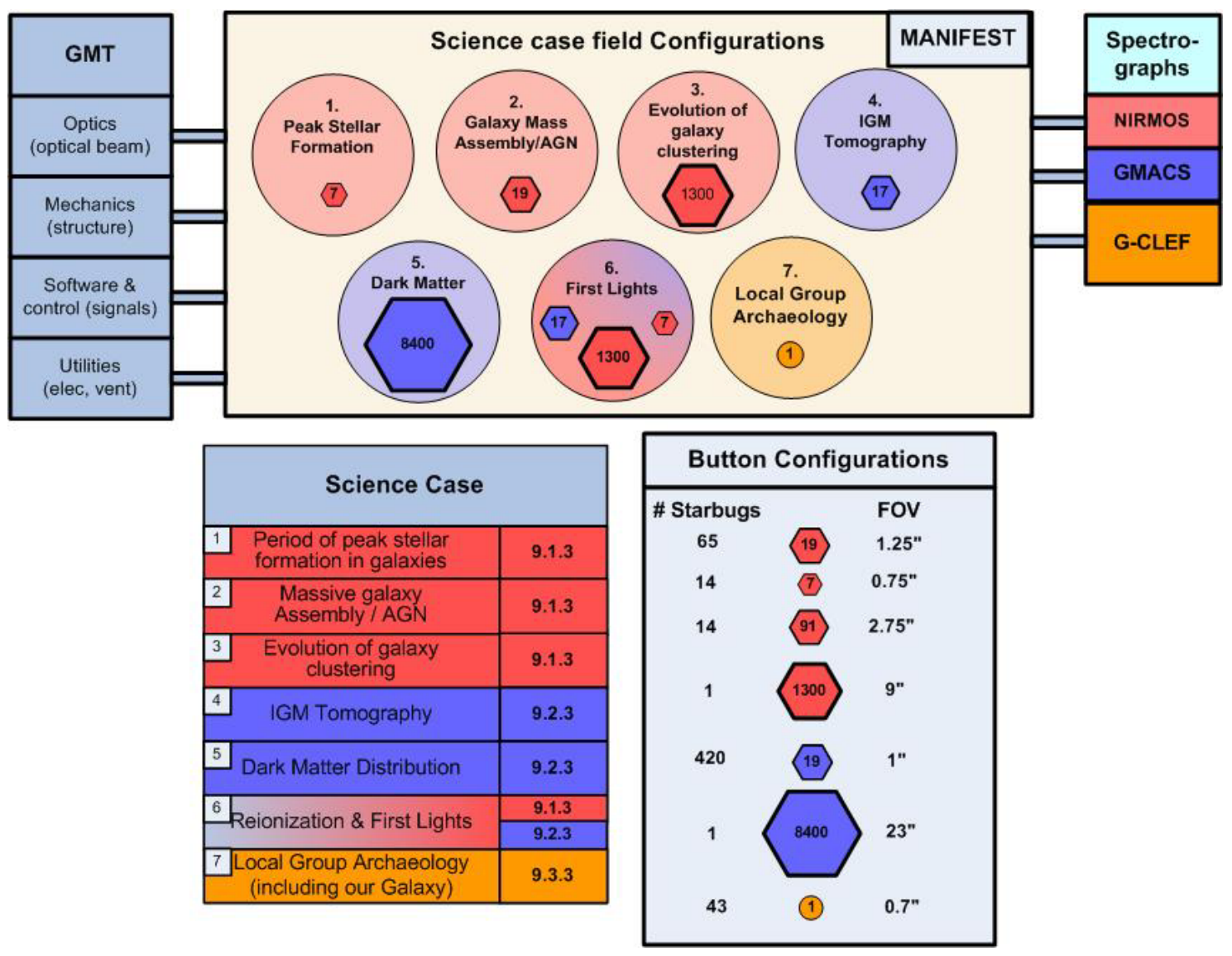

Figure 1: MANIFEST capabilities and GMT science goals

\section{INSTRUMENT CONCEPT}

\subsection{Positioning Concept}

The MANIFEST instrument concept is based on a system of semi-autonomous walking probes called "Starbugs" 10, 11, 12 that hold and position hundreds of optical fiber IFUs under a glass field plate placed at the GMT Cassegrain focal plane (see Figure 2). The Starbug probes feature co-axial piezoceramic tubes, which via the application of appropriate AC waveforms, contract or bend, providing a discrete stepping motion. Simultaneous positioning of all Starbugs (allowing full field configuration in a few minutes) is achieved via a closed-loop metrology system. In the baseline design, a vacuum between the piezoceramic tubes is used to keep the Starbugs in place by suction for any orientation of the telescope.

As part of the MANIFEST Feasibility Study a fully functional Starbugs demonstrator system (Figure 4) was fabricated featuring interchangeable glass field plates, a high-resolution metrology camera, and full control electronics for up to 20 Starbugs. This system has been demonstrated to meet the rather exacting requirements in terms of parallel control, positioning accuracy, configuration speed and Starbug pitch. 


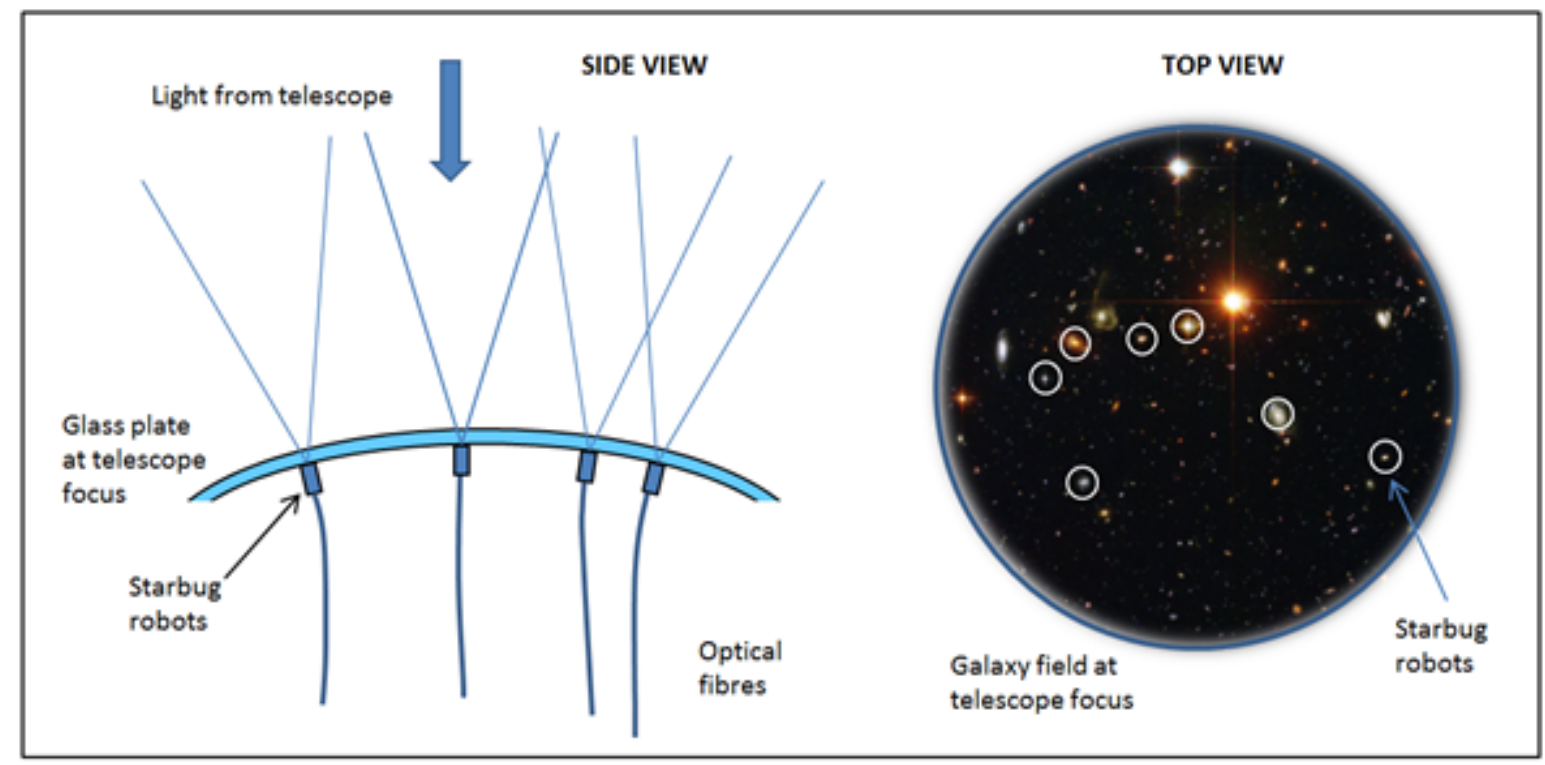

Figure 2: Schematic view of MANIFEST starbugs and field plate.

\subsection{Fore- and Post-Optics}

Most MANIFEST IFUs operate through image-slicing. Their fore-optics (see Figure 4) consists of a pair of silica microlens arrays inside the Starbug tubes, facing each other, with the fibers cemented to the substrates of the lower micro-lens. They image multiple pupil images on the fibers with telecentric beams. Fibre bundles are routed to the instrument entrance slits. Fibre connectors are used to allow maintenance, and instruments to be moved or removed. The MANIFEST post-optics mirror the fore-optics as closely as possible. For the image-slicing modes, pupil-imaging at output allows a slit filling factor of almost unity, with only small gaps between the spectra (caused by the small gaps between adjacent lenslets). Since adjacent spectra are unresolved on the sky, and will be accurately lined up spectrally, the resulting crosstalk is minimal.

The fiber slits will be made up of individual slitlets, attached to a mounting plate. NIRMOS and G-CLEF slitlets will have thin prisms bonded to them to achieve the required exit angles to become pupil-centric within these spectrographs.

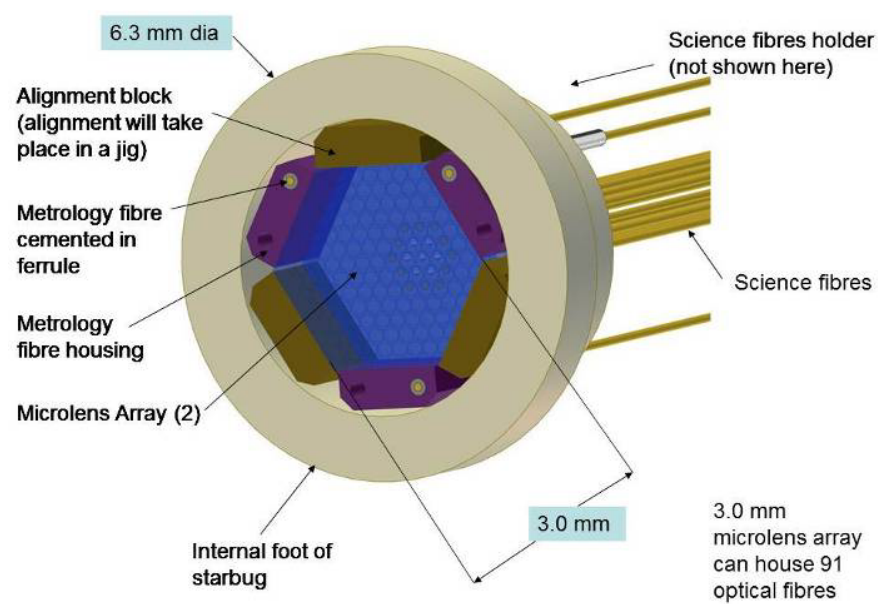

Starbug Fore Optics Conceptual Design

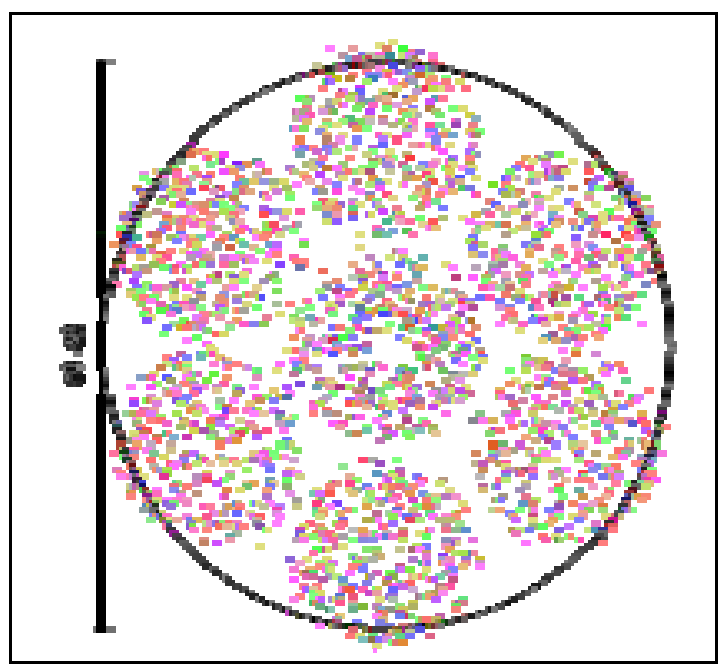

IFU GMT pupil image overfilling a $89 \mu \mathrm{m}$ core fiber

Figure 3: Fore -optics design for Starbug. Left: Starbug Fore Optics Conceptual Design. Right: IFU GMT pupil image overfilling a $89 \mu \mathrm{m}$ core fiber 


\subsection{Probe Configuration}

Analysis of the prime science cases for MANIFEST led to the selection of six different probe sets, each feeding the entrance slit (or slits) of one GMT instrument. Table 3 and Figure 4 show the basic parameters of each of these modes. There are also up to 19 Starbug guide probes (2.5" FoV) coupled to a video camera and 75 back-illuminated fiducial fibers at the edge of the $20^{\prime}$ field for probe metrology and configuration.

The Glass Field Plate (GFP) holds also a fixed active optics probe either right at the field center, or possibly making an equilateral triangle with the NIRMOS and GMACS IFUs. Preliminary specs are for a 15" diameter acquisition field and a 5" diameter active optics field. This capability is part of the Telescope Control System and will help in accurate field acquisition and focusing.

Table 3: Baseline probe configurations

\begin{tabular}{|c|c|c|c|c|c|c|c|}
\hline Set & Mode & \# Probes & $\begin{array}{l}\text { Probe } \\
\text { fiber \# }\end{array}$ & Sampling & Field $\Phi$ & $\begin{array}{c}\text { Patrol field } \Phi \\
\quad \text { (global) }\end{array}$ & $\begin{array}{l}\text { Patrol field R } \\
\text { (individual) }\end{array}$ \\
\hline N1 & $\begin{array}{l}\text { Survey } \\
\text { Mode }\end{array}$ & 65 & 19 & $0.25^{\prime \prime}$ & $1.25^{\prime \prime}$ & $17.5^{\prime}$ & $3.5^{\prime}$ \\
\hline $\mathrm{N} 2$ & $\begin{array}{l}\text { Mass } \\
\text { assembly } \\
\text { Sky probes }\end{array}$ & $\begin{array}{l}14 \\
14\end{array}$ & $\begin{array}{c}91 \\
7\end{array}$ & $0.25^{\prime \prime}$ & $\begin{array}{l}2.75^{\prime \prime} \\
0.75^{\prime \prime}\end{array}$ & $7.0^{\prime}$ & GLAO field \\
\hline N3 & $\begin{array}{l}\text { OH- } \\
\text { suppression }\end{array}$ & 1 & 19 & $0.125^{\prime \prime}$ & $0.625^{\prime \prime}$ & Fixed & Fixed \\
\hline N4 & Single IFU & 1 & 1300 & $0.25^{\prime \prime}$ & $9^{\prime \prime}$ & Fixed & Fixed \\
\hline $\mathrm{GM}^{+}$ & $\begin{array}{l}\text { Survey } \\
\text { mode }\end{array}$ & 420 & 19 & $0.25^{\prime \prime}$ & $1.25^{\prime \prime}$ & $20.0^{\prime}$ & $3.5^{\prime}$ \\
\hline $\mathrm{GM}^{+}$ & Single IFU & 1 & 8400 & $0.25^{\prime \prime}$ & $23^{\prime \prime}$ & Fixed & Fixed \\
\hline $\mathrm{GC} 1$ & $\begin{array}{l}\text { Multi- } \\
\text { object }\end{array}$ & $43 / 4$ & 1 & $0.7^{\prime \prime}$ & $0.7^{\prime \prime}$ & $20.0^{\prime}$ & $3.5^{\prime}$ \\
\hline
\end{tabular}

Col. 1: reference code (N: NIRMOS, GM: GMACS , GC: G-CLEF); Col. 2: observing mode; Col. 3: number of probes; Col. 4: number of fibers per probe; Col. 5: field sampling; Col. 6: probe field diameter; Col. 7: accessible field diameter covered by all probes; Col. 8: patrol field radius covered by each probe.

+ Note: GM1 \& GM2 modes assume a 4 arm GMACS configuration.

\subsection{Focal Plane Assembly}

MANIFEST is fed by GMT equipped with its Wide-Field Corrector (WFC), with a clone of the last WFC lens (L3) attached to MANIFEST. This optical system delivers a $1250 \mathrm{~mm}\left(20^{\prime}\right)$ diameter corrected convex F/8.5 telecentric focal surface with a $3275 \mathrm{~mm}$ radius of curvature (ROC). MANIFEST's $\sim 3 \mathrm{~mm}$ thick GFP is positioned on the focal surface. The on-sky scale is $1.0^{\prime \prime} / \mathrm{mm}$. The exit pupil is at the center of curvature of the focal surface, ensuring that the probes' optical axes are automatically aligned with the F/8.5 beams. The telescope can deliver instead a ground layer adaptive optics (GLAO) corrected field of 7' $(420 \mathrm{~mm})$ diameter with similar properties. 


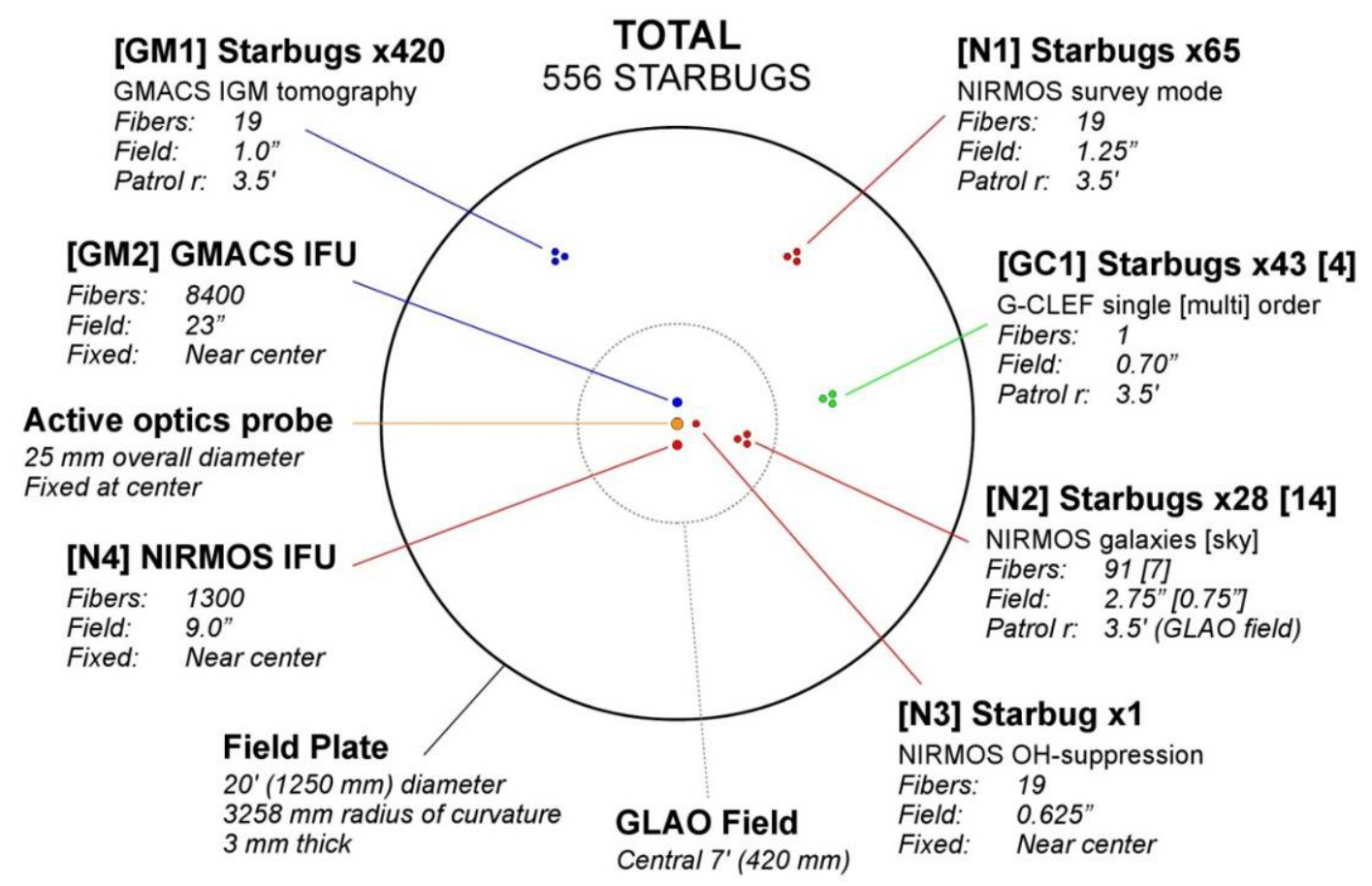

Figure 4: Baseline probe deployment

\subsection{Mechanical Design}

MANIFEST is installed in the Gregorian Instrument Rotator (GIR), sharing one of the four spaces provided for instrumentation with one pair of GMACS spectrographs. It is mounted under GMACS in an Instrument Mounting Frame (IMF), manufactured to meet MANIFEST-specific requirements. The overall size of the IMF will allow its installation and removal from the GIR.
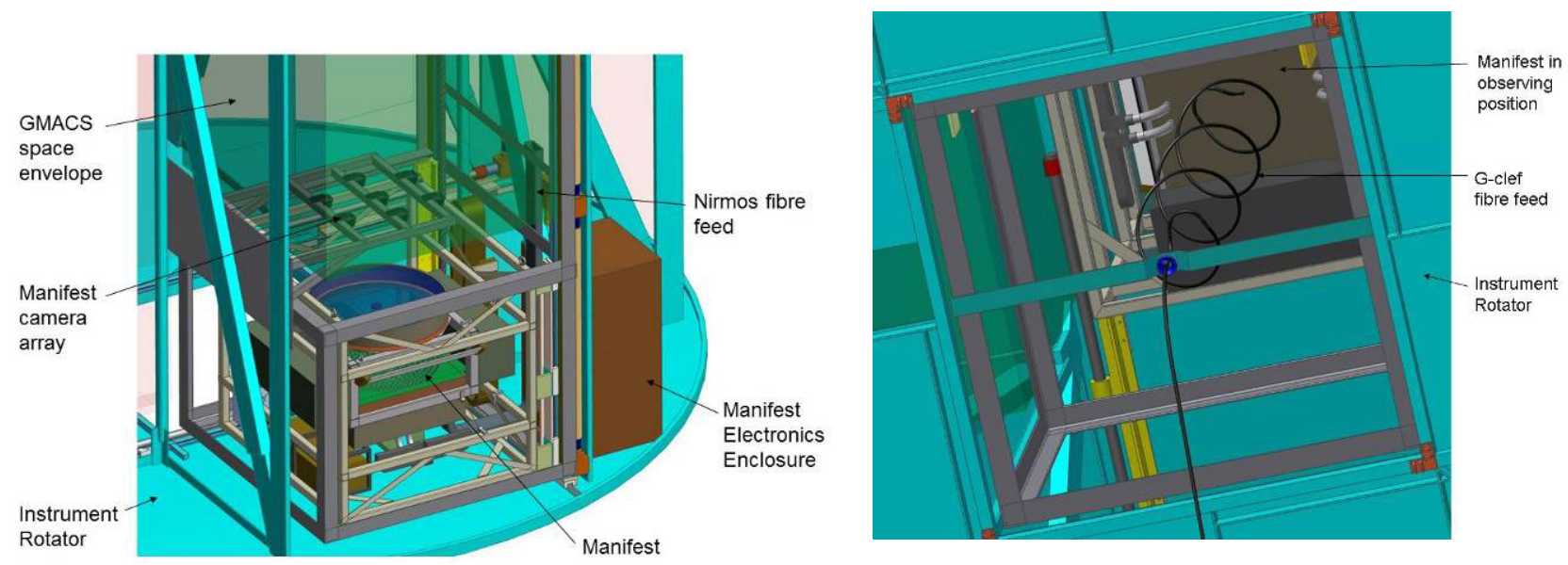

Figure 5: MANIFEST in its 'storage' position (left) and the fiber feed for G-CLEF (right) 
For operation, MANIFEST moves inwards and up in the GMT central field. A kinematical reference, preferably close to the upper vertex of L3, will ensure a good repeatability, likely of $\pm 1 / 4 \mathrm{~mm}$ or better in all three axis.

MANIFEST is made of two modules, the internal and external frames shown respectively in Figure 6. The internal frame holds the Field Lens and Field Plate Unit and a Connector/Harness Plate. The Field Lens/Plate Assembly is supported both around its perimeter and with a central post that holds the active optics system required by GMTO. The internal frame is enclosed by transparent panels to create a dust-free environment.

Fibers and wires run from the Starbugs and the fixed IFUs to a connector plate $500 \mathrm{~mm}$ below the field plate. The length of the fiber and electric bundles is $\sim 10 \%$ oversized to allow the Starbugs to "patrol". The fibers and electrodes terminate in a connector plate to create a modular system to simplify installation and maintenance.

The internal frame is connected to the external frame by four pairs of tendons. The external frame carries the electronics enclosures, the power supply system, the $\mathrm{OH}$-suppression grating fibers and the Calibration Camera Array. Most of the electronics equipment is located between the Instrument Mounting Frames within the GIR.
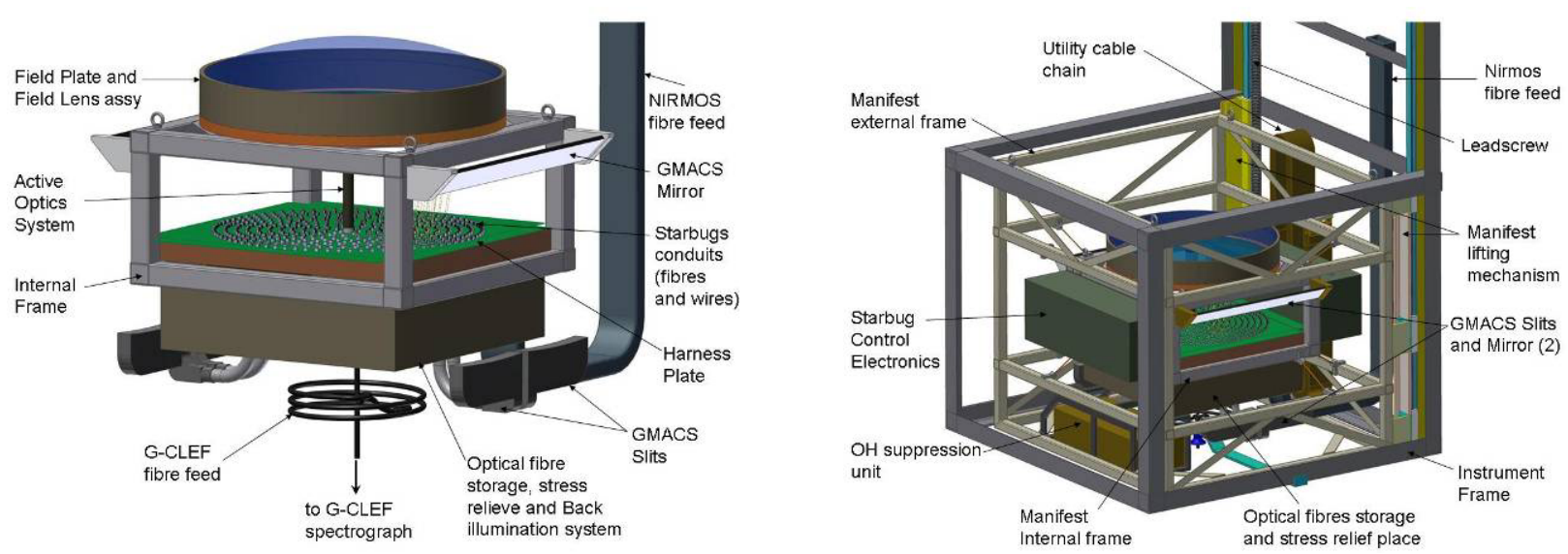

Figure 6: MANIFEST - internal (left) and external (right) frame sub-system

\subsection{Metrology Concept}

Accurate positioning of the probes relative to the telescope focal plane is critical to the operation and performance of any multi-fiber spectrographic facility. A dedicated metrology system is thus required whenever probes are selected and configured on the GFP for subsequent science observing. The principle is to image the back-illuminated fiber probes with a video camera and move the probes to the required positions under closed-loop operation.

The goal is to limit to at most $2 \%$ the light losses coming from 3 -axis positioning errors and resulting PSF degradation for a $0.75^{\prime \prime}$ diameter FoV probe under median seeing conditions $\left(0.65^{\prime \prime}\right)$. On the metrology side, this translates in achieving $20 \mu \mathrm{m} \mathrm{rms} \mathrm{Starbug} \mathrm{coordinate} \mathrm{accuracy} \mathrm{over} \mathrm{the} \mathrm{full} 1250 \mathrm{~mm}$ diameter field plate. This leaves a $75 \mu \mathrm{m} \mathrm{rms}$ error budget for repositioning errors of the glass field plate on the GMT focal plane.

Moreover, this accurate probe positioning must be achieved in a few minutes in order not to waste telescope time. The metrology facility must be available both with MANIFEST in its parked position (for engineering purposes) and when it is deployed at the focal plane (for science operations). It should have minimal impact on telescope resources and operations, and preferably be modular and use off-the-shelf components.

The proposed metrology architecture uses two separate systems for science and engineering purposes. The science system is made of four (single when available off the shelf) cameras with lenses mounted at the GMT M2 assembly to image the field plate. The engineering system uses a camera array located above the MANIFEST parked position to ensure $100 \%$ availability. It is permanently fixed in the GIR, $1.2 \mathrm{~m}$ above the focal plane. It also holds a fish-eye lens for inspection of the full field. 
Each Starbug holds three back-illuminated fibers in an asymmetric pattern external to its science field. They serve as metrology fiducial points for precise position measurement, with on/off lighting for probe identification. Field centers are determined by triangulation of the back-illuminated fibers, with the offsets precisely measured on each Starbug during MANIFEST's Assembly, Integration \& Testing (AIT).

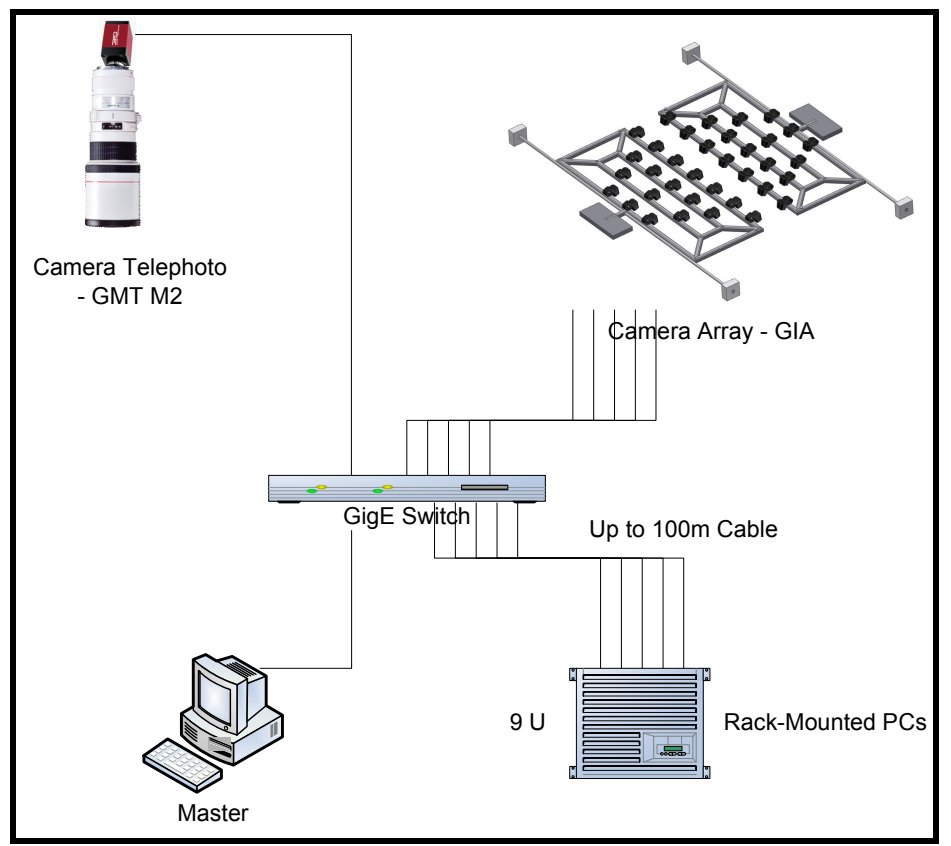

Figure 7: Metrology Architecture

Registration of the metrology cameras with respect to the glass field plate is achieved using 75 LEDs glued on the infocus inner side of the field plate and regularly distributed on a circle just outside the $1250-\mathrm{mm}$ diameter science field. The multiple camera fields of each science and engineering metrology systems overlap by at least $20 \%$. This ensures that the LED fiducials located on the field plate perimeter and back illuminated Starbugs in the overlapped regions provide sufficient information for registration of the whole field. This frees up the need for inner LED fiducials, which would otherwise interfere with the field plate environment.

Efficient gathering of light from science objects into probe fibers over the large MANIFEST field requires a high metrological stability of the Telescope/WFC plus MANIFEST system. This is not easy with an active/adaptive ELT and a multi-ton instrument that moves in and out of its working position.

A nominal alignment error budget goal of $75 \mu \mathrm{m} \mathrm{rms}$, which is divided into $53 \mu \mathrm{m}$ rms for telescope scale change and 53 $\mu \mathrm{m}$ rms for MANIFEST misalignment effects. On the GMT side, this translates to a modest requirement on the stability of the telescope plate scale, between successive repositionings, of $8.5 \times 10^{-5}$. MANIFEST does not require this scale to be constant, only that it is known and sent by the Telescope Control System to the MANIFEST positioning software. The $53 \mu \mathrm{m} \mathrm{rms}$ allowance for individual Starbug positions is split into a metrology error (which should be $<20 \mu \mathrm{m}$ ), and actual positioning rounding errors, which should not amount to more than a few microns. 
Table 4: Science Metrology Camera System Specifications

\begin{tabular}{|l|l|l|}
\hline Specification & Value & Description \\
\hline Location & M2 $(20 \mathrm{~m}$ above F/8 focus $)$ & clear view of full field plate \\
\hline Field sampling & $4000 \times 4000$ pixels & requires 4 off the shelf cameras \\
\hline Pixel scale & $315 \mu \mathrm{m}$ at F/8 focus & centroid accuracy $1 / 20$ pixel for S/N 30 \\
\hline Frame rate & $>15 \mathrm{fps}$ & Starbug motion tracking up to $7 \mathrm{~mm} / \mathrm{s}$ \\
\hline Exposure & $<10 \mathrm{~ms}$ & mitigate differential motions \\
\hline Imaging & Telephoto F/5.6 Lens & $400 \mathrm{~mm}$ focal length for $6 \mu \mathrm{m}$ CCD pixel \\
\hline Interface & GigE Ethernet & standard interface; up to $100 \mathrm{~m}$ cable run \\
\hline Unit size & $150 \times 150 \times 500 \mathrm{~mm}$ & minimal impact of mounting on M2 \\
\hline Cameras & $4 \times$ Prosilca GE2040 & $\begin{array}{l}2040 \times 2040,6 \mu \mathrm{m} \text { pixel, } 15 \mathrm{fps}, \mathrm{CCD} \\
20 \% \text { field overlap }\end{array}$ \\
\hline
\end{tabular}

MANIFEST moves both laterally and axially on motorized rails from its parked to observing position. A kinematical reference tied to the GIR frame, preferably close to the 1st surface of L3, will ensure that MANIFEST always goes back to the same observing position: a $\sim 250 \mu \mathrm{m}$ accuracy in the three axis is required and should be reasonably achieved.

\section{TECHNOLOGY RESEARCH AND DEVELOPMENT}

The AAO has a long-term R\&D program aimed at developing new techniques and technologies that can be used to enhance the performance, efficiency, and cost-effectiveness of astronomical instrumentation. This research program has several key aspects: robotic positioning systems, fiber technologies, and astrophotonics. In this section, we outline the key technological R\&D that has been conducted at the AAO that specifically relates, or is of relevance, to the MANIFEST instrument. This includes work that was conducted as part of the MANIFEST feasibility study, and work that has been conducted in parallel to this study.

\subsection{Starbug Prototyping}

Starbugs ${ }^{10,11,12}$ are the parallel fiber positioning devices of MANIFEST. AAO is conducting an extensive R\&D program to prepare this technology for astronomical applications. Starbugs are discrete stepping devices, featuring two co-axial piezoceramic tubes joined at their top ends; see Figure 8 (b). The application of a voltage across the wall of a piezoceramic tube will cause it to expand, contract, or bend. The magnitude of the tube's movement depends on the magnitude of the voltage applied: a displacement of the order of tens of microns is typical for a potential of several hundred volts. By driving a Starbug's two co-axial tubes with specific AC waveforms, a stepping motion can be achieved. Starbugs adhesion to the glass field plate is achieved with a vacuum between the inner and outer piezoceramic tubes. Starbugs performances over all field plate angle from 0 (zenith) to 90 degrees (horizon) are encouraging with controllable speeds from 0 to $5 \mathrm{~mm} / \mathrm{s}$ and step-sizes from 3 to 18 microns, depending on the drive frequency and voltages. The Starbug's mass is approximately 2-3 grams. Starbug to Starbug minimum pitch has been reduced from $16 \mathrm{~mm}$ to about $8 \mathrm{~mm}$ (respectively 16 " and 8 " on the sky at GMT) with the use of vacuum adhesion to the field plate, instead of the previous system relying on magnetic forces. A recent development includes adding controlled rotation with an angular step of about 4 arcminutes. 


\subsection{Starbugs Demonstrator}

Development and testing of Starbugs technology has been facilitated by a fully functional demonstrator, shown in Figure 8 (a). The demonstrator features interchangeable glass field plates (including curved plates), a high-resolution metrology camera, and full control electronics for up to 20 Starbugs in parallel. The system enclosure is mounted on an axle, allowing computer-controlled rotation of the entire system up to $\pm 90^{\circ}$ from zenith. The control system interfaces with a host computer running Starbugs control software and enables automated testing of Starbug performance under controlled conditions. The demonstrator has been successfully used to demonstrate a 10-vacuum starbug system with closed loop positioning over a $400 \mathrm{~mm}$ field plate to $1 / 20$ pixel (approx. 10 microns) at field plate angles of 0 (zenith) and 90 degrees (horizon).

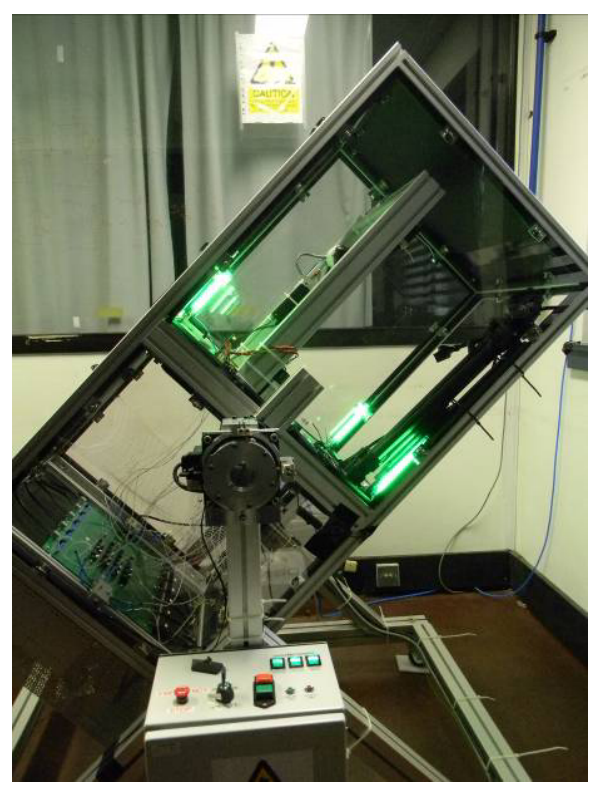

(a) Test-rig demonstrator unit
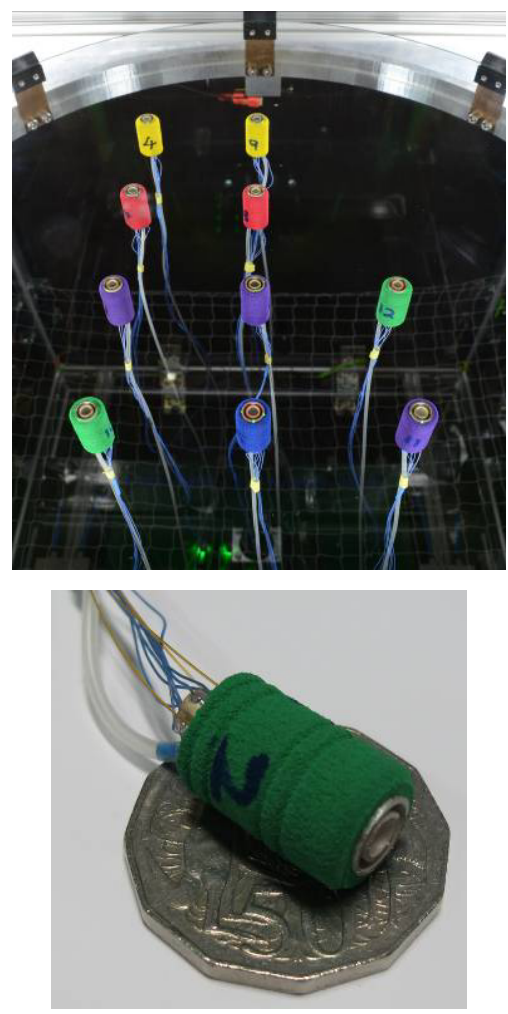

(b) Starbug devices

Figure 8: Starbugs R\&D - positioning devices for MANIFEST

\subsection{Glass Field Plate}

During the feasibility study we developed a preliminary specification for the field plate, prototyped a version fabricated via one relatively low cost method (slumping, see Figure 9 and Figure 10), and obtained rough order of magnitude costing for fabrication via more sophisticated methods and for coating to our specification. A detailed tolerancing analysis has now lead to a firmer optical specification (see Figure 11) in terms of surface irregularity, bubble inclusion (surface close to focal plane) and plate tolerances limited by the vignetting within the fore optics. Based on the new optical specification we have been in communication with three optical suppliers and workshops, each using a different method of fabrication for the field plate. A further understanding of the processes, costs, and time frames involved has been achieved. The old magnet clamped design ${ }^{4,11}$ required a low-friction 'Teflon' coating on the upper side of the large field plate that was difficult to achieve. The new vacuum clamped one does not require it anymore and we are discussing with vendors to get firm cost and performance estimates for the now relatively standard coatings. 

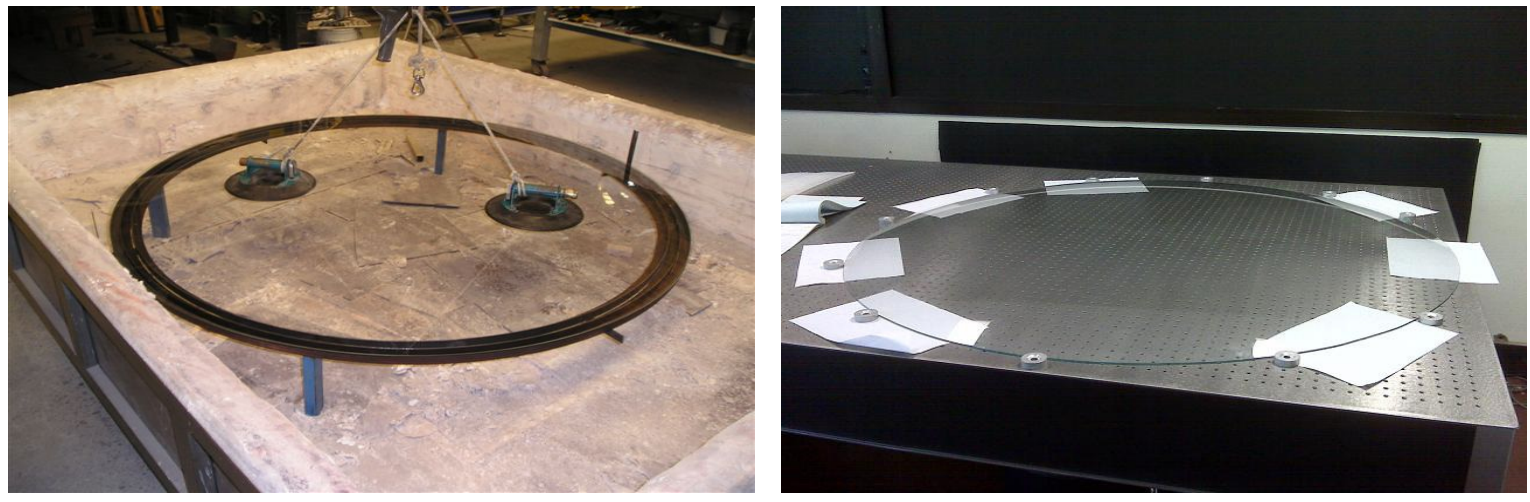

Figure 9: Prototype 1.3 m diameter Starphire GFP; (left) in furnace; (right) metrology test
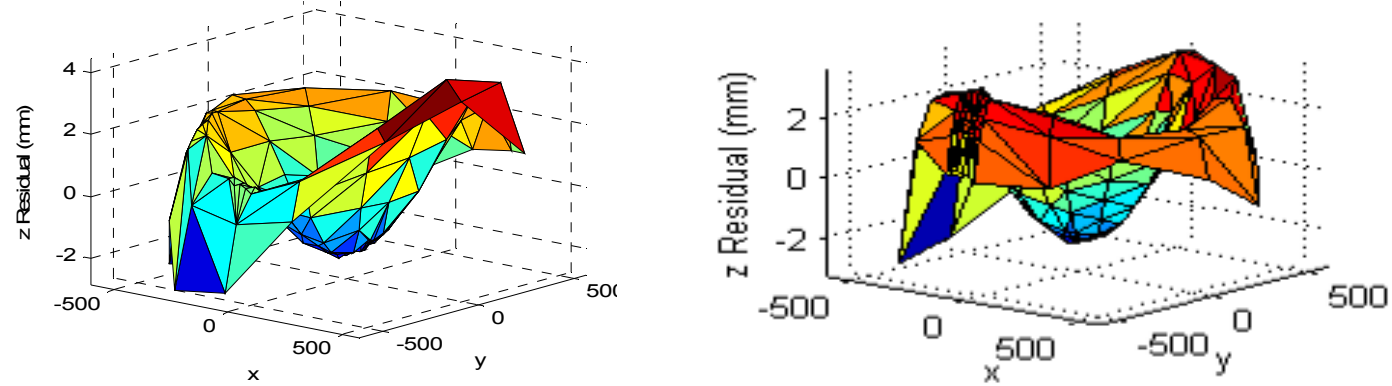

Figure 10: RMS z-residuals slumped 1.3m diam. test plates: (L) $3 \mathrm{~mm}$ (R) \& 4mm thickness

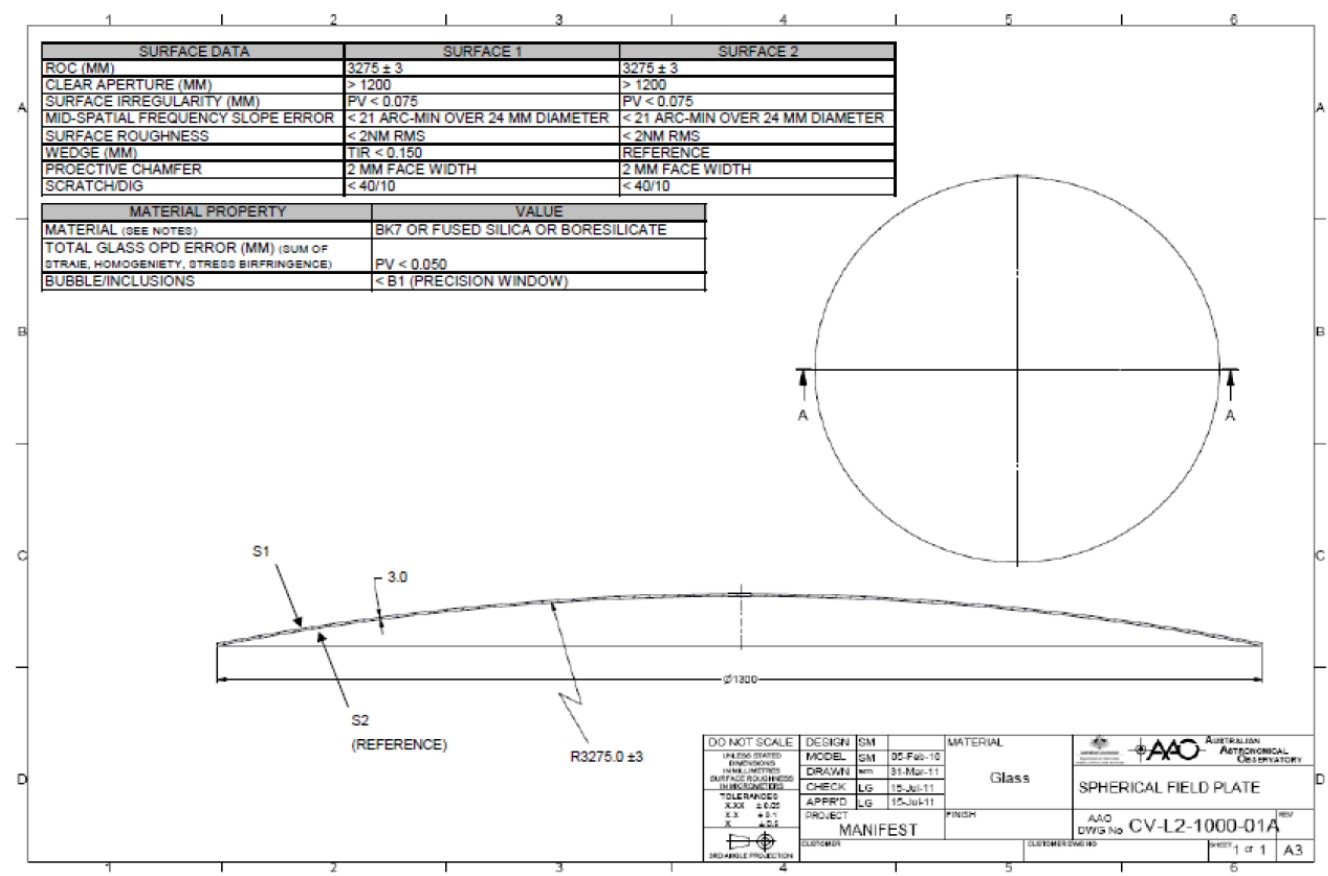

Figure 11: Field plate specification drawing 


\subsection{Fiber Technologies \& Astrophotonics}

The AAO in collaborations with the University of Sydney and Macquarie University, has an ongoing R\&D efforts into fiber related technologies and astrophotonic devices for astronomical applications. These devices include (a) hexabundles $^{13}$; (b) fiber tapers ${ }^{14}$; (c) fiber vacuum feedthroughs; (d) fiber connectors and suitable fibers ${ }^{15}$; (e) fiber design; and (f) photonic $\mathrm{OH}$ suppression ${ }^{16,17}$. We are continuing to develop these technologies and will consider their use in the MANIFEST instrument as further prototyping studies are completed.

\section{PERFORMANCE}

This section provides preliminary MANIFEST throughput and observing mode parameters that can be incorporated into the Exposure Time Calculators (ETC) of NIRMOS, GMACS and G-CLEF. This data enables the quantitative analysis of how MANIFEST complements the scientific potential of these three instruments. The throughput addresses the whole MANIFEST optical path starting with the Glass Field Plate and ending with light injection into the NIRMOS, GMACS, and G CLEF slits. The results are summarized in Figure 12. It must be noted that as a feasibility study, the performances and specifications are subject to change as MANIFEST is further developed.

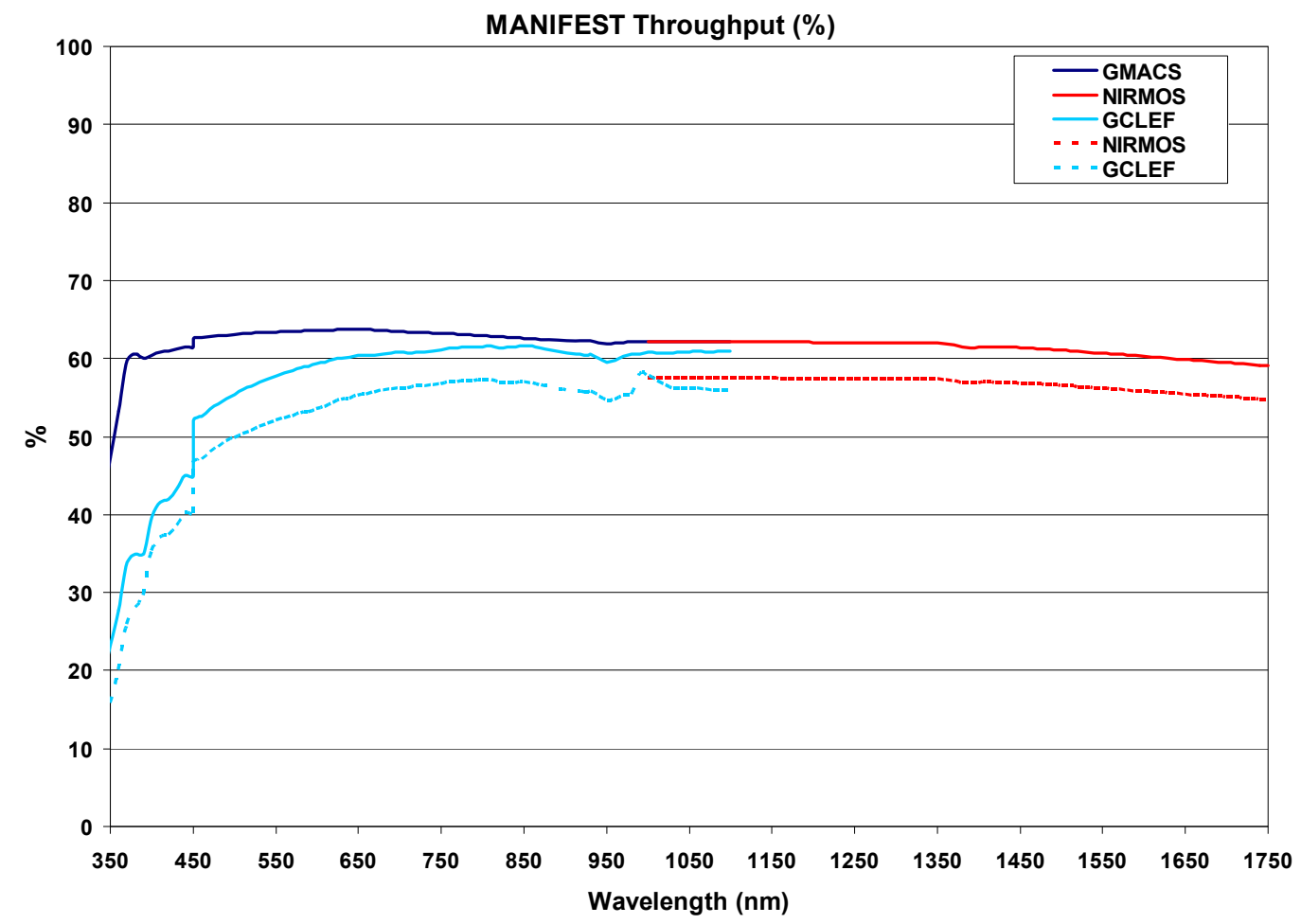

Figure 12: MANIFEST-only throughput as a function of wavelength for each instrument

Figure 12 shows the MANIFEST-only throughputs as functions of wavelength. The blue solid curve applies to GMACS, the cyan solid curve to G-CLEF, and red solid curve to NIRMOS. The major source of loss is the fore-optics component, approximately $\sim 25 \%$. Since GMACS standalone and GMACS fed by MANIFEST share the same WFC/ADC ${ }^{18}$, the solid blue curve also represents their differential throughput. In the G-CLEF and NIRMOS cases, only MANIFEST is using respectively the WFC/ADC and the WFC/Dummy-ADC (for NIRMOS), and the dashed cyan and red curves show the respective differential throughputs. The roughly factor 2 difference in transmission at $350 \mathrm{~nm}$ between the GMACS and G-CLEF cases is due to the large difference in fiber length, viz. $5 \mathrm{~m}$ versus $25 \mathrm{~m}$. 


\section{CONCLUSIONS}

The MANIFEST Feasibility Study has a) retired the primary technology risks through design and prototyping studies, b) determined the functional and scientific added value of the baseline instrument design, c) estimated the cost to construction for both the baseline system and various descope/staging options, and d) identified, and built into the baseline design, the essential requirements for the interface with the telescope and the other instruments. In addition, the long-term R\&D program led by the AAO has, since the completion of the MANIFEST Feasibility Study, further advanced the technology readiness of key instrument components, with in addition significant performance gains.

MANIFEST will further help GMT to become a world-leading facility over a wide swath of astronomy, providing both new capabilities and substantial gains in observational efficiency for survey work. We have demonstrated that the key technologies required for MANIFEST are all viable, and that this facility would be a cost-effective addition to the GMT instrument suite.

\section{ACKNOWLEDGEMENTS}

Australian membership in the GMT consortium is funded by the Department of Innovation, Industry, Science and Research, and Tertiary Education (DIISRTE) through the Australian Government Education Investment Fund (EIF). The MANIFEST Feasibility Study was funded by EIF, the Giant Magellan Telescope Organization (GMTO) and the National Science Foundation under Scientific Program No. 10 as issued for support of the Giant Segmented Mirror Telescope for the United States Astronomical Community, in accordance with Proposal No. AST-0443999 submitted by AURA.

\section{REFERENCES}

[1] Lewis, I.J., Cannon, R.D., Taylor, K., Glazebrook, K., Bailey, J.A., Baldry, I.K., Barton, J.R., Bridges, T.J., Dalton, G.B., Farrell, T.J., Gray, P.M., Lankshear, A., McCowage, C., Parry, I.R., Sharples, R.M., Shortridge, K., Smith, G.A., Stevenson, J., Straede, J.O., Waller, L.G., Whittard, J.D., Wilcox, J.K. and Willis, K.C., "The Anglo-Australian Observatory 2dF facility," MNRAS 333, 279-299 (2002).

[2] Gillingham, P.R., Popovic, D., Farrell, T. J. and Waller, L.G., "The performance of OzPoz, a multi-fiber positioner on the VLT”, Proc SPIE 4841, 1170-1179 (2003).

[3] Shectman, S., Johns, M., "GMT overview", Proc. SPIE 7733, 77331Y (2010).

[4] Saunders, W., Colless, M., Saunders, I., Hopkins, A., Goodwin, M., Heijmans, J., Brzeski, J., and Farrell, T., "MANIFEST: a many-instrument fiber-positioning system for GMT", Proc. SPIE 7735, 773568 (2010).

[5] The Giant Magellan Telescope Project, "GMACS - The GMT Wide-Field Optical Spectrograph", GMT Conceptual Design Report, Section 13.6, http://www.gmto.org/codrfolder/GMT-ID-01478-Section_13.6_GMACS.pdf (2006).

[6] The Giant Magellan Telescope Project, "Near Infrared Multiple Object Spectrograph", GMT Conceptual Design Report, Section 13.5, http://www.gmto.org/codrfolder/GMT-ID-01477-Section_13.5_NIRMOS_.pdf (2006). 
[7] Szentgyorgyi, A., Charbonneau, D., Latham, D., Sasselov, D. and Torres, G., "The GMT-CFA Large Earth Finder (G-CLEF): A high stability optical echelle spectrograph for exoplanet and cosmology research" , http://www.slidefinder.net/s/stgeorgi_gclef/9458705 (2010).

[8] Thirty Meter Telescope , "Wide-Field Optical Spectrometer (WFOS)", http://www.tmt.org/sites/default/files/WWW_WFOS_DRF01.doc (2009).

[9] Le Fèvre, O., Hill, L., Le Mignant, D., Maccagni, D., Tresse, L., Paltani, S., "OPTIMOS-DIORAMAS: A Wide-field Imaging and Multi-slit Spectrograph for the E-ELT.”, The Messenger, vol. 140, p. 34-35

[10] McGrath, A. and Moore, A., "Starbug: enabling the smart focal plane”, Proc. SPIE 5495 600-610 (2004).

[11] Goodwin, M., Heijmans, J., Saunders, I’, Brzeski, J., Saunders, W., Mueller, R., Haynes, R., Gilbert, J., “Starbugs: focal plane fiber positioning technology”, Proc. SPIE 7739, 77391E (2010)

[12] Gilbert, J., Goodwin, M., Heijmans, J., Mueller, R., Miziarski, S, Brzeski, J., Waller, L., Saunders, W., Bennet, A., Tims, J,. "Starbugs: all-singing, all-dancing fibre positioning robots", Proc. SPIE 8450-44, in press.

[13] Bland-Hawthorn, J. et al., "Hexabundles: imaging fibre arrays for low-light astronomical applications", Proc. SPIE 7735 , in press.

[14] Marcel, J., Haynes, R., Bland-Hawthorn, J., “Application of fiber tapers in astronomy”, Proc. SPIE 6273, 62733X (2006).

[15] Polymicro Technologies,, http://www.polymicro.com/products/opticalfibers/products_opticalfibers_fbp.htm

[16] Bland-Hawthorn J., Englund M., Edvell, G., "New approach to atmospheric OH suppression using an aperiodic fibre Bragg grating.”, Opt.Express 12(24), 5902-9 (2004).

[17] Ellis, S.C., Bland-Hawthorn, J., "The case for OH suppression at near-infrared wavelengths”, MNRAS 333, 279 299 (2002).

[18] The Giant Magellan Telescope Project , “Optics”, GMT Conceptual Design Report, Chapter 6, http://www.gmto.org/codrfolder/GMT-ID-01467-Chapter_6_Optics.pdf (2006).

[19] Gillingham, P.R., Miziarski,S., Akiyama,M. and Klocke, V., "Echidna: a multi-fiber positioner for the Subaru prime focus", Proc. SPIE 4008, 1395-1403 (2000). 